ISAHP Article: A Style Guide for Paper Proposals To Be Submitted to the International Symposium of the Analytic Hierarchy Process 2016, London, U.K.

\title{
The identification of adequate control structure for AHP and ANP
}

*Note: Do not include the author(s) names and information as this document will be blind reviewed and they will be entered during proposal submission.

\section{ABSTRACT}

The reliability of AHP and ANP analysis results depends on the application of a structure that adequately describes relations between a decision problem model components. Model structure adequacy is especially important in the case of modeling complex systems with possible dependence and feedback between their components. But even in the case of problems related to complex systems structures of their models are usually constructed in a rather subjective manner. The structures are also often simplified to facilitate analysis of the systems. Fortunately, there are also decision making problem model structuring techniques available which make finding an adequate system model structure possible in a less subjective manner. The paper deals with their application to provide adequate AHP and ANP control structures while solving decision making problems.

Keywords: AHP, ANP, result, reliability, model, structure, identification.

\section{Introduction}

The utilization of a proper structure for a model of a given decision making problem is crucial for the reliability of AHP and ANP analysis outcomes. An adequate structure of relations between problem model components for a decision making problem under consideration should be therefore carefuly identified. Hopefully there are some decision problem model structuring approaches available which provide necessary means to identify an adequate problem model structure in a less subjective manner.

\section{Literature Review}

Literature review reveals a number of decision making problem model structuring approaches. For example, Fontela \& Gabus (1976) proposed the application of DEcision MAking Trial and Evaluation Laboratory (DEMATEL) in this regard. On the other hand Warfield (1974) proposed the Interpretative Structural Modeling (ISM). Both approaches are capable of identifying key decision making problem model components and the key relations which link model components. Note that especially DEMATEL seems an ideal complement to ANP. This is because it delivers necessary means for confirming the interactions between model components and information about such interactions for a successful ANP application. That is why several ways for complementary application of DEMATEL and ANP are proposed (Golcuk \& Baykasoglu 2016).

International Symposium on the Analytic Hierarchy Process 


\section{Hypotheses/Objectives}

The direct and indirect application of a structuring approach may provide a structure for a decision making problem model. However level of structure complexity may make the structure infeasible for being used as a control structure needed for AHP/ANP. The structure undergoes therefore a necessary reduction. It seems, however, that the simplification level should be controled to avoid inadequacy of an oversimplified model of a decision making problem.

\section{Research Design/Methodology}

A procedure is proposed to deal with the problem of adequate simplification of a decision making problem model structure. It makes use of both exisiting problem structuring approaches and an own problem structuring approach. The application of some auxiliary means is also applied to assure adequacy of obtained reduced structure (Ginda 2015) .

\section{Data/Model Analysis}

Results of a sample analysis are presented to illustrate merits of the proposed procedure. Data from literature are applied to make calculations.

\section{Limitations}

A sample analysis confirms merits of the proposed procedure. The procesure seem to provide efficient means for limiting negative influence of subjective decisions on adequacy of a simplified structure of a decision making problem. The procedure requires a further and more thorough justification. Some improvements are also possible. For example procedure may be enhanced by developing more robust criteria for the quality of a decision making problem structure reduction level.

\section{Conclusions}

The importance of applied control structure adequacy is often underestimated while using AHP/ANP. A need for the simplification of a control structure to make a decisioon problem tractable makes the problem even more important. Therefore the proposed procedure makes seems a vital step towards the reliable identification of an adequate structure for a decision making problem model under consideration.

\section{Key References}

Fontela, E., \& Gabus., A. (1976), DEMATEL Observer. DEMATEL 1976 Report. Batelle Research Institute, Geneva, Switzerland.

Warfield, J.N. (1974), Toward interpretation of Complex Structural Models, IEEE Transactions on Stystems, Man, Cybernetics, SMC-4(5):405-417.

Golcuk, I., Baykasolglu, A. (2016). An analysis of DEMATEL approaches for criteria interaction handling within ANP. Expert Systems with Applications 46:346-366.

Ginda, G. (2015). Pair-wise comparison-based methods for decision making support in civil engineering and related fields. DWE, Wrocław. 